\title{
Self-healing Janus Interfaces for High-performance LAGP-based Lithium Metal Batteries
}

Qi Liu',2, Dong Zhou ${ }^{3,}{ }^{,}$, Devaraj Shanmukaraj', Peng Lí, Feiyu Kang', Baohua $L i{ }^{1,{ }^{*},}$, Michel Armand ${ }^{4,}$, , Guoxiu Wang ${ }^{3,{ }^{*}}$

${ }^{1}$ Shenzhen Key Laboratory on Power Battery Research and Shenzhen Geim Graphene Center, School of Shenzhen International Graduate, Tsinghua University, Guangdong 518055, China;

${ }^{2}$ School of Materials Science and Engineering, Tsinghua University, Beijing 100084, China

${ }^{3}$ Centre for Clean Energy Technology, Faculty of Science, University of Technology Sydney, NSW 2007, Sydney, Australia.

${ }^{4}$ CIC ENERGIGUNE, Parque Tecnológico de Álava, Miñano 01510, Spain

${ }^{5}$ College of Material Science and Engineering, Nanjing University of Aeronautics and Astronautics, Nanjing, 210006, P.R. 


\section{EXPERIMENTAL SECTION}

\section{Preparation of LAGP pellet}

The $\mathrm{Li}_{1.5} \mathrm{Al}_{0.5} \mathrm{Ge}_{1.5}\left(\mathrm{PO}_{4}\right)_{3}$ (LAGP, $500 \mathrm{~nm}$ ) raw powder was purchased from Hefei kejing Material Technology Co., Ltd. The LAGP electrolyte pellet was synthesized by pressing the powder into pellets (thickness: $\sim 300 \mu \mathrm{m}$; diameter: $12 \mathrm{~mm}$ ), and then sintering at $850{ }^{\circ} \mathrm{C}$ for $12 \mathrm{~h}$ under air atmosphere in muffle furnace.

\section{Preparation of the SHEs}

The UPyMA monomer was synthesized through a previously reported method. ${ }^{1}$ To prepare the ALE, $0.5 \mathrm{M}$ bis(trifluoromethane)sulfonimide lithium salt (LiTFSI, $>99.0 \%$ DoDoChem) was first dissolved into (1-ethyl-3-methylimidazolium bis(trifluoromethylsulfonyl)imide, EMITFSI, $\geq 97.0 \%$, Sigma-Aldrich) ionic liquid, followed by adding $10 \mathrm{wt} \%$ ethylene carbonate (EC, $>99.0 \%$, DoDoChem) as additive into above mixture. $1 \mathrm{M}$ LiTFSI was dissolved in adiponitrile (AN, $99.5 \%$, Aladdin) reagent to obtain the CLE. To prepare the ASHE/CSHE, $3 \mathrm{wt} \%$ UPyMA monomer was first dissolved into the ALE or CLE at $60{ }^{\circ} \mathrm{C}$, respectively. Subsequently, $1.5 \mathrm{wt} \%$ pentaerythritol tetraacrylate (PETEA, 98\%, Sigma-Aldrich) as crosslinker and $0.1 \mathrm{wt} \%$ 2-hydroxy-2-methylpropiophenone (HMPP, 97\%, Sigma-Aldrich) as initiator were added into the mixture to obtain transparent ASHE/CSHE precursor solution. After that, the precursor solution was dropped on the LAGP pellet, and then exposed to ultraviolet (UV)-irradiation from an Hg UV lamp (with an irradiation peak intensity of $\sim 2000 \mathrm{~mW} \mathrm{~cm}{ }^{-2}$ ) for $15 \mathrm{~min}$ to 
ensure a fully polymerization of the monomers. As a result, a thin ASHE/CSHE interlayer with a thickness $\sim 10 \mu \mathrm{m}$ was in-situ constructed on the LAGP pellet. The AGPE was prepared by polymerizing $1.5 \mathrm{wt} \%$ PETEA and $0.1 \mathrm{wt} \%$ HMPP in ALE. Meanwhile, the CGPE was prepared by polymerizing $1.5 \mathrm{wt} \%$ PETEA and $0.1 \mathrm{wt} \%$ HMPP in CLE. All above procedures were prepared in an argon-filled glove box (Universal 2440/750) with a moisture/oxygen concentration below $0.1 \mathrm{ppm}$.

To separate and purify the polymer matrixes from the ASHE/CSHE, the polymer electrolytes were mashed into pieces and repeatedly washed with acetone, followed by collecting the white precipitate with filtration. Finally, the white precipitate was dried at $120{ }^{\circ} \mathrm{C}$ for $24 \mathrm{~h}$ in a vacuum oven to obtain the separated ASHE/CSHE polymer matrixes.

\section{Characterization of the SHEs}

Fourier transform infrared spectra (FTIR) were recorded by a Bruker Vertex70 instrument at room temperature. For the combustion test, $0.1 \mathrm{~g}$ electrolyte samples were prepared and then the optical photographs and videos were captured after the samples were ignited. The weight losses of ASHE, CSHE and commercial liquid electrolyte (1 $\mathrm{M} \mathrm{LiPF}_{6}$ in EC: $\mathrm{DEC}(1: 2$ by volume) $)$ were recorded by aging at $25^{\circ} \mathrm{C}$ in the open air. To evaluate the thermal stability of electrolyte samples, thermogravimetric analysis (TGA) measurements were carried out from room temperature to $150{ }^{\circ} \mathrm{C}$ with an $\mathrm{N}_{2}$ flow at a rate of $5{ }^{\circ} \mathrm{C} \mathrm{min}-1$ on a Mettler Toledo TGA thermoanalyzer. The ionic conductivities of electrolyte samples were measured from 
$25{ }^{\circ} \mathrm{C}$ to $95{ }^{\circ} \mathrm{C}$ by electrochemical impedance spectroscopy (EIS) on a VMP3 multichannel electrochemical station (Bio Logic Science Instruments, France) with small alternating current amplitude of $10 \mathrm{mV}$ from $10 \mathrm{mHz}$ to $7 \mathrm{MHz}$. The investigated cells were assembled by sandwiching a piece of electrolyte sample between two stainless steel blocking electrodes, and keeping at each testing temperature for $30 \mathrm{~min}$ before the EIS test. For the LAGP, conductive silver paste was coated on the LAGP surface before the ionic conductivity tests to ensure good contacts to the stainless steel|LAGP interfaces. Linear sweep voltammetry (LSV) curves were recorded to study the electrochemical stability of the investigated electrolytes. The assembled coin cells were tested from the open circuit potential to 5 $\mathrm{V}\left(v s . \mathrm{Li} / \mathrm{Li}^{+}\right)$at a scanning rate of $1 \mathrm{mV} \mathrm{s}^{-1}$ by the VMP3 electrochemical station, where stainless steel blocking electrode was used as the working electrode and Li foil was used as both the counter and reference electrodes.

Galvanostatic cycling measurement was conducted to investigate the compatibility of ASHE interface layer with Li metal anode, which was performed on a symmetric Li|ASHE|LAGP|ASHE|Li cell at $0.1 \mathrm{~mA} \mathrm{~cm}^{-2}$ with a cut-off capacity of $0.1 \mathrm{mAh}$ $\mathrm{cm}^{-2}$. The Li electrode after 10 cycles was rinsed with DEC (Sigma-Aldrich) in argon-filled glove box, and then transferred into in-depth X-ray photoelectron spectroscopy (XPS, PHI 5000 VersaProbe $I I$ spectrometer with a monochrometer Al $\mathrm{K} \alpha \mathrm{X}$-ray source at $1486.6 \mathrm{eV}$ ) and atomic force microscope (AFM, Brucker Dimension Icon) tests. The Young's modulus of the Li metal surface was estimated based on the force-indentation curve using RTESPA-150 tip, which was obtained by 
the analysis of the Nanoscope Analysis software. X-ray diffraction (XRD) patterns of LAGP solid electrolyte were collected for the phase identification after the cycling using a Rigaku D max 2500 diffractometer with $\mathrm{Cu} \mathrm{K} \alpha$ radiation $(\lambda=1.5418 \AA)$. The average Coulombic efficiency $\left(C E_{a v g}\right)$ of Li plating and stripping was conducted in the a $\mathrm{Li} \| \mathrm{Cu}$ cell configuration using method reported by Zhang et al. ${ }^{2}$ The $\mathrm{Cu}$ substrate was preconditioned with one Li deposition/stripping cycle with a capacity of $1 \mathrm{mAh} \mathrm{cm}^{-2}$ to depositing the $\mathrm{Li}$ reservoir $\left(Q_{T}\right)$. After that, the cell was discharged-charged with a small capacity of $0.1 \mathrm{mAh} \mathrm{cm}^{-2}\left(Q_{C}\right)$ for $n$ cycles, followed by a final exhaustive strip of the remaining Li reservoir to $1 \mathrm{~V}$. The final stripping charge $\left(Q_{S}\right)$, corresponding to the quantity of Li remaining after cycling, is measured. The average CE over $n$ cycles can be calculated as:

$C E_{a v g}=\frac{n Q_{C}+Q_{S}}{n Q_{C}+Q_{T}}$

The Li deposition morphology was characterized by field emission scanning electron

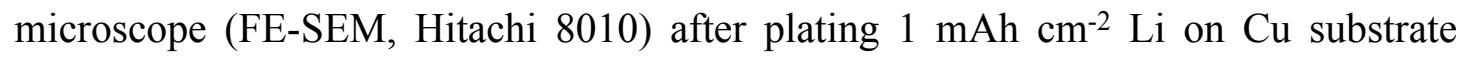
under $0.1 \mathrm{~mA} \mathrm{~cm}^{-2}$.

\section{Characterization of the solid-state $\mathrm{Li}|| \mathrm{LMO}$ metal batteries}

All of the LAGP-based solid-state cells were fabricated with $\mathrm{LiMn}_{2} \mathrm{O}_{4}(\mathrm{LMO}$, Shanshan Co., Ltd) as the cathode, thin Li foil as the anode and LAGP or as treated-LAGP as the solid electrolyte without any separators. The LMO active material, super $\mathrm{P}$ and polyvinylidene fluoride $(\mathrm{PVdF})$ binder were grinded together in a mass ratio 80: 10: 10 in anhydrous N-methyl-2-pyrrolidone (NMP, Sigma-Aldrich). 
The slurry was subsequently coated on an aluminium current collector and dried overnight at $70{ }^{\circ} \mathrm{C}$. The mass loading of the obtained cathode was $\sim 1.2 \mathrm{mg} \mathrm{cm}$. Electrochemical properties were evaluated with CR2032 coin cells by a Land 2001 A battery testing system, which were assembled in an argon-filled glovebox with a moisture/oxygen concentration below $0.1 \mathrm{ppm}$. The integrated Li||LMO full cells were charged and discharged between $2.8 \mathrm{~V}$ and $4.3 \mathrm{~V}$ vs. $\mathrm{Li} / \mathrm{Li}^{+}$. The cycling performances were collected at various rates $\left(1 \mathrm{C}=148 \mathrm{~mA} \mathrm{~g}^{-1}\right.$ based on the mass of LMO) at room temperature. For the Li|LAGP|LMO cell configuration, a little amount of commercial liquid electrolyte ( $\sim \mathrm{uL}$ of $1 \mathrm{M} \mathrm{LiPF}_{6}$ in EC: DEC (1: 2 by volume)) was dropped on the cathode side to ensure the interfacial wetting. EISs of the Li $\|$ LMO cells after certain cycle were measured on the VMP3 electrochemical workstation with alternating current amplitude of $10 \mathrm{mV}$ from $100 \mathrm{mHz}$ to $1 \mathrm{MHz}$.

\section{Theoretical calculations}

All the calculations of adsorption energy were carried out using ORCA 4.2.0 package. ${ }^{3}$ Both of geometry optimization and single point energy were calculated with $\omega B$ 97M-D3BJ method. ${ }^{4-5}$ The basis sets of def2-SVP and def2-TZVP 6 were employed in geometry optimization and single point energy, respectively. 


\section{List of Supporting Figures}
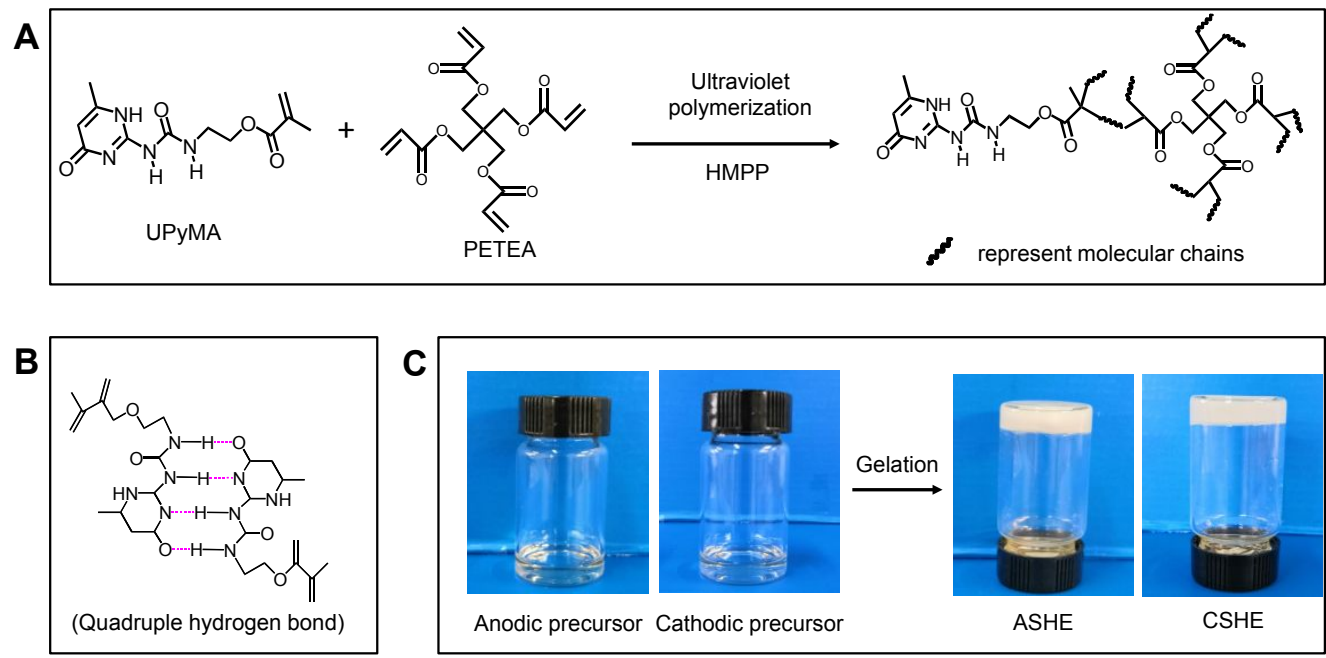

Figure S1. (A) The reaction mechanism to synthesis the SHE. (B) Schematic illustration of quadruple hydrogen bond among UPyMA monomers. (C) The optical images of the gelation processes from the precursor solution to SHEs under UV irradiation. 


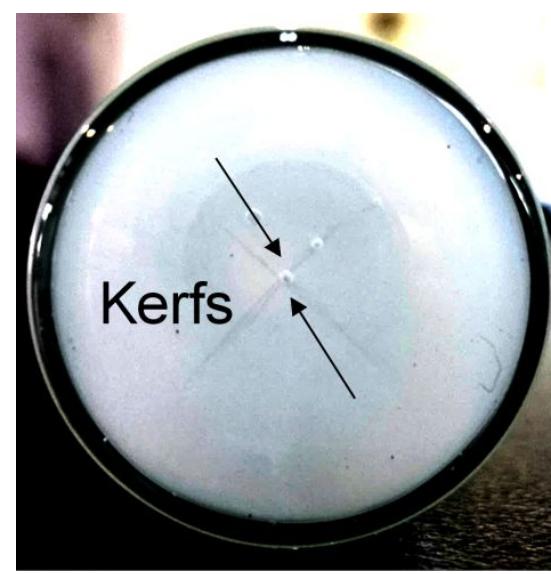

$24 \mathrm{~h}$ aging

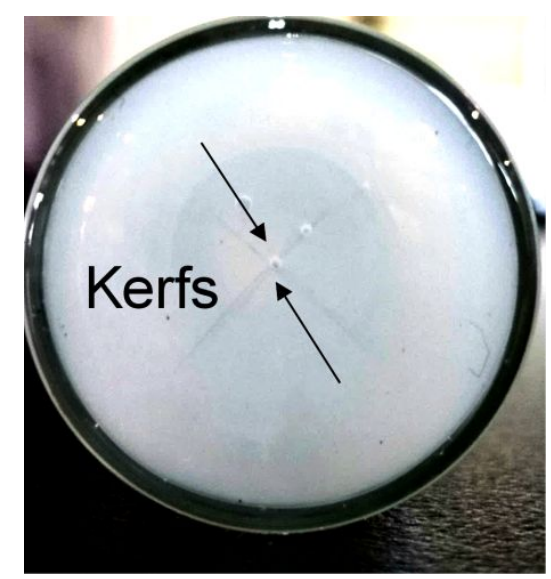

Figure S2. Optical images of the self-healing processes of AGPE after being cut. 


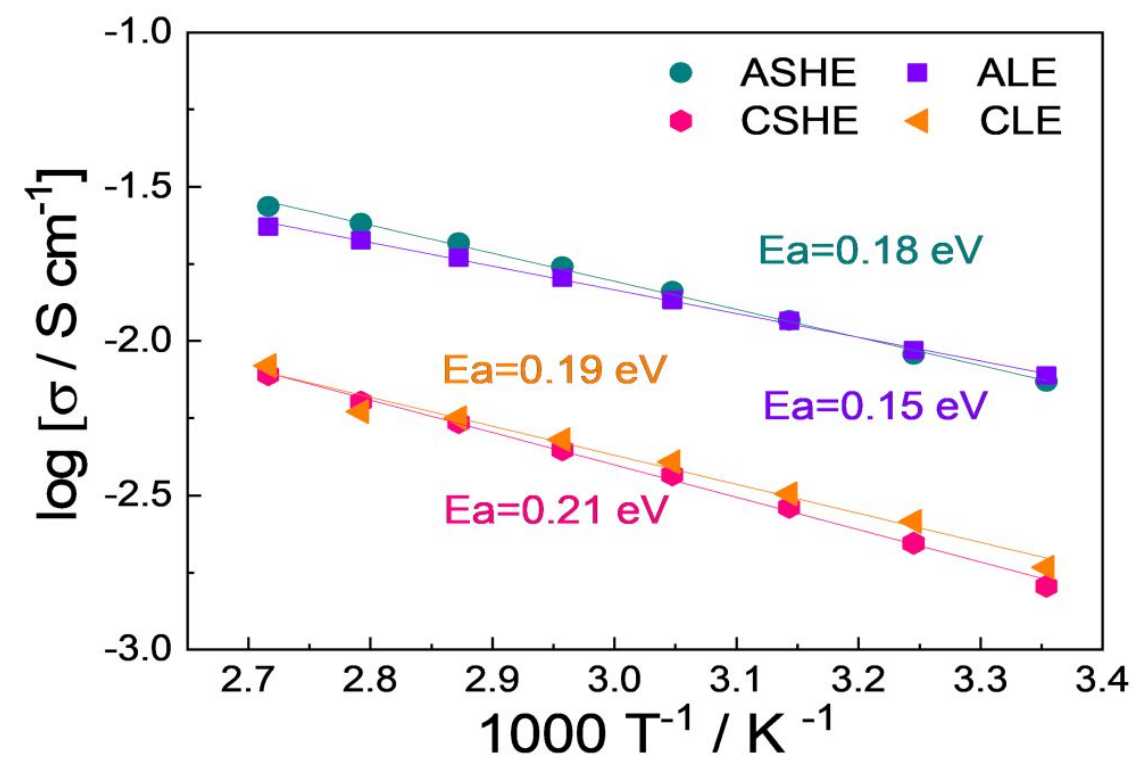

Figure S3. The ionic conductivities of ASHE, CSHE, ALE and CLE as a function of temperature. The plots represent the experimental data meanwhile the solid lines represent Arrhenius equation fitting results. 


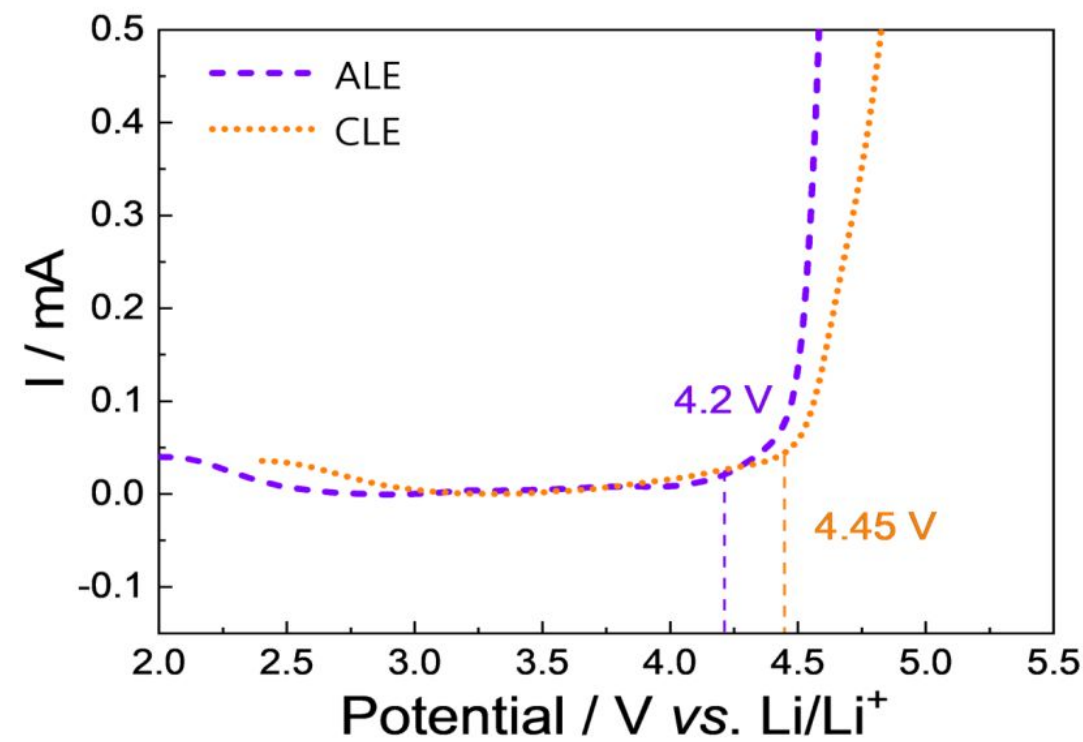

Figure S4. LSVs of ALE and CLE at a scan rate of $1 \mathrm{mV} \mathrm{s}^{-1}$. 


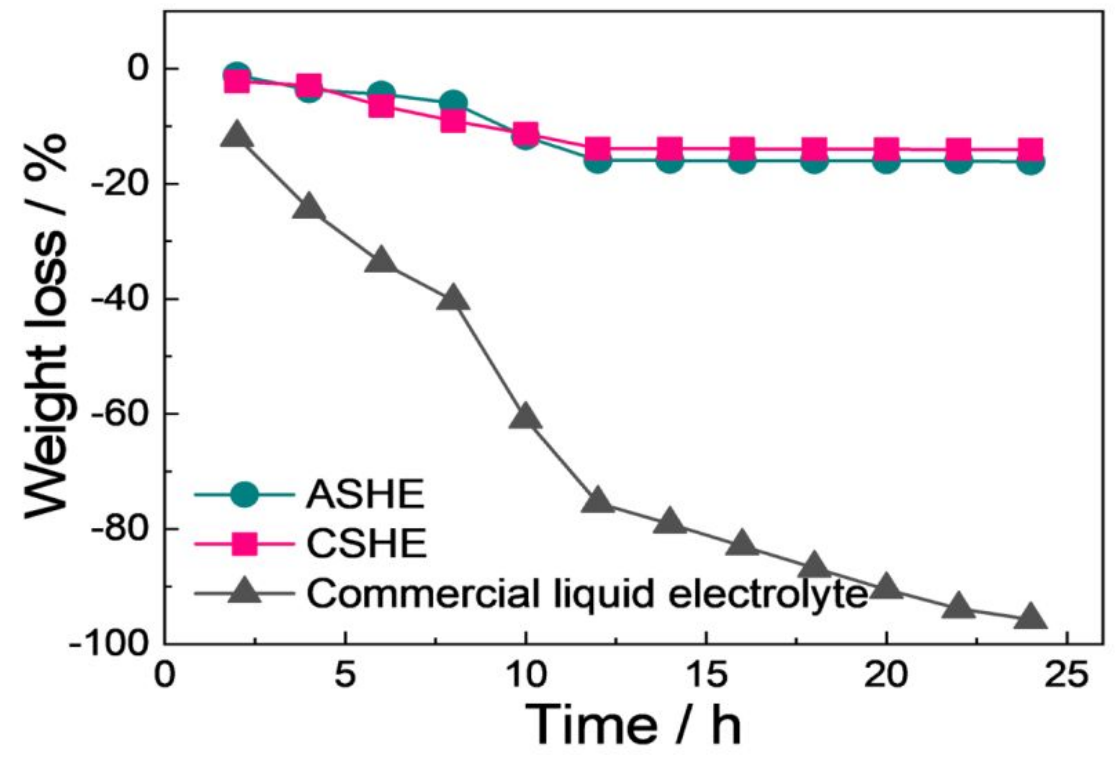

Figure S5. The weight losses of the $1 \mathrm{M} \mathrm{LiPF}_{6}$ in EC: DEC (1: 2 by volume) commercial liquid electrolyte, ASHE and CHE during an aging of $24 \mathrm{~h}$ at $25^{\circ} \mathrm{C}$. 


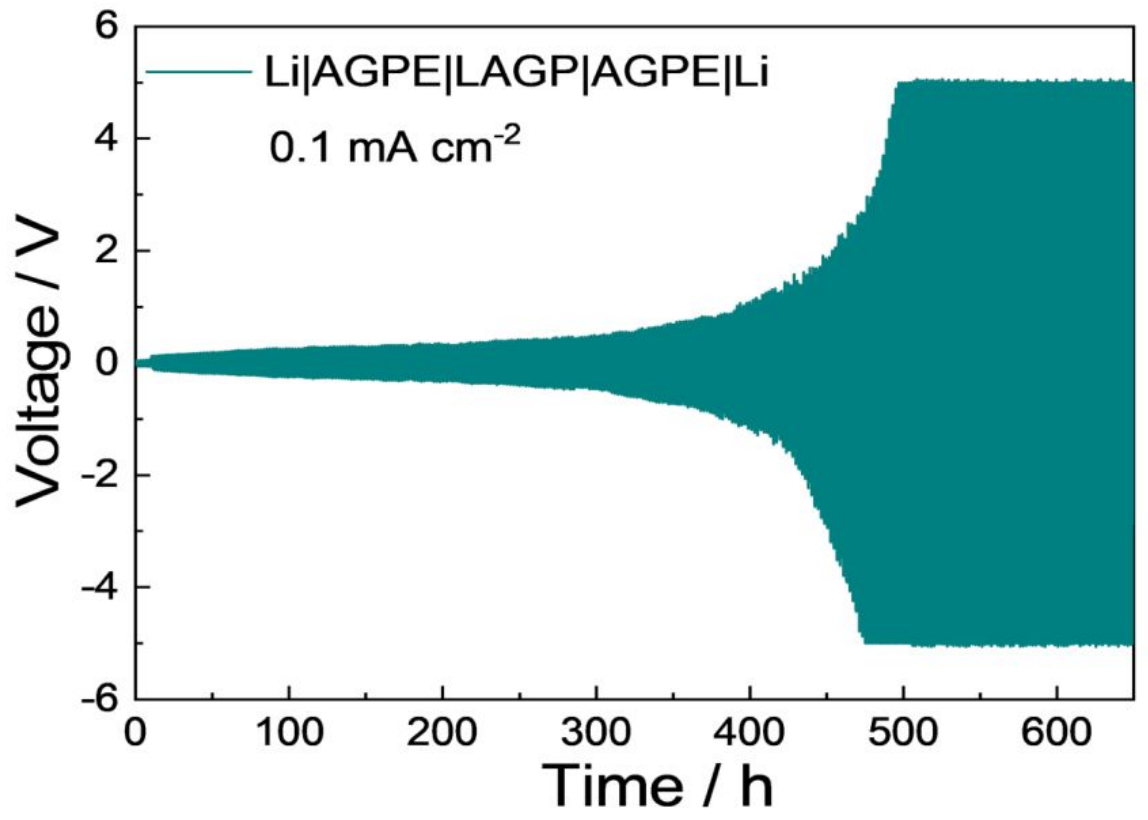

Figure S6. Galvanostatic cycling curves of Li|AGPE $\mid$ LAGP $\mid$ AGPE $\mid$ Li symmetrical cells at $0.1 \mathrm{~mA} \mathrm{~cm}{ }^{-2}$ with a cut-off capacity of $0.1 \mathrm{mAh} \mathrm{cm}^{-2}$. 


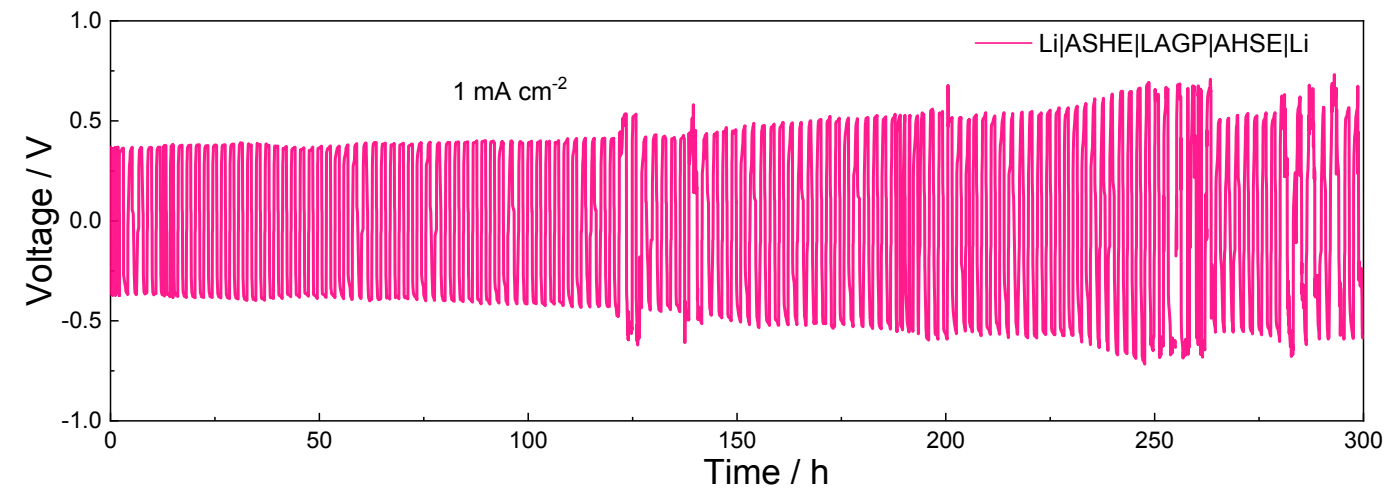

Figure S7. Galvanostatic cycling curve of Li|ASHE|LAGP|ASHE|Li symmetrical cells at $1 \mathrm{~mA} \mathrm{~cm}^{-2}$ with a cut-off capacity of $1 \mathrm{mAh} \mathrm{cm}^{-2}$. 


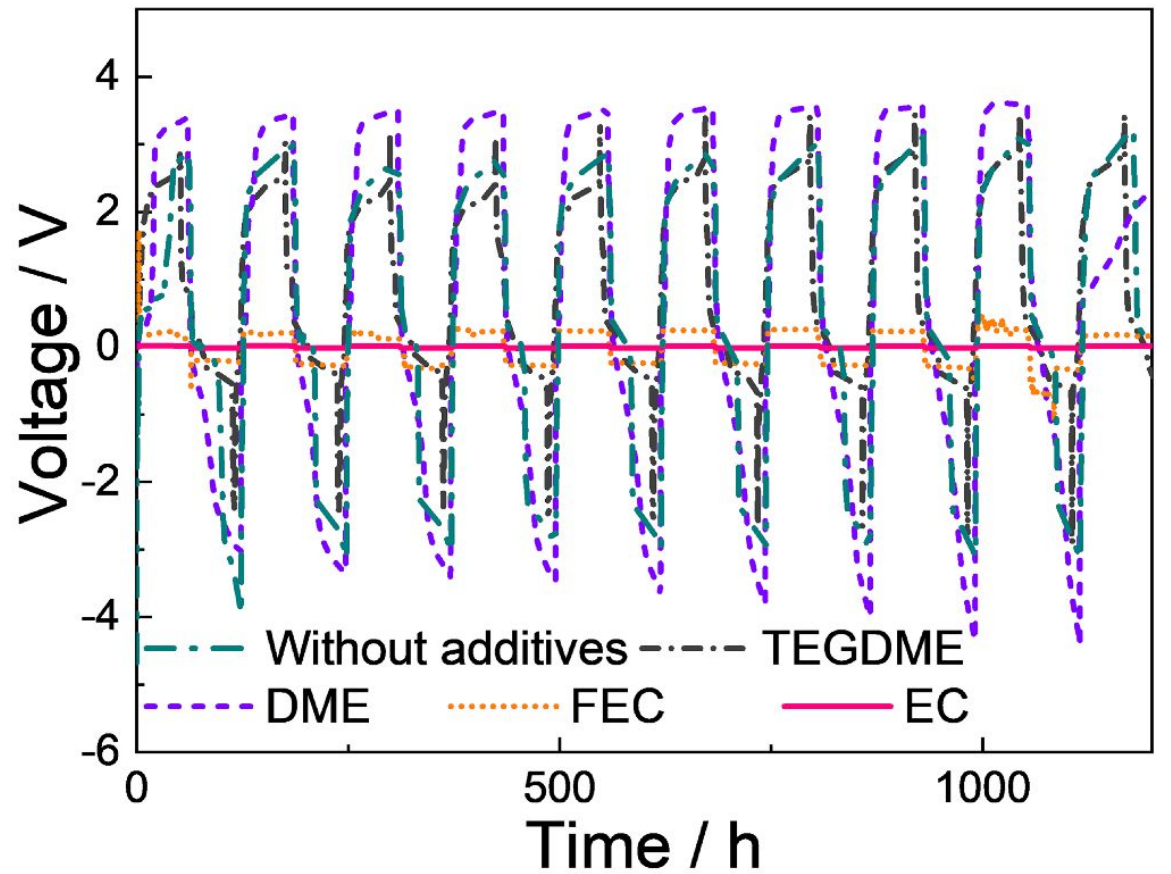

Figure S8. Galvanostatic cycling curves of Li|ASHE|LAGP|ASHE|Li cells with different electrolyte additives at $0.1 \mathrm{~mA} \mathrm{~cm} \mathrm{~cm}^{-2}$ with a cut-off capacity of $0.1 \mathrm{mAh} \mathrm{cm}{ }^{-2}$. 

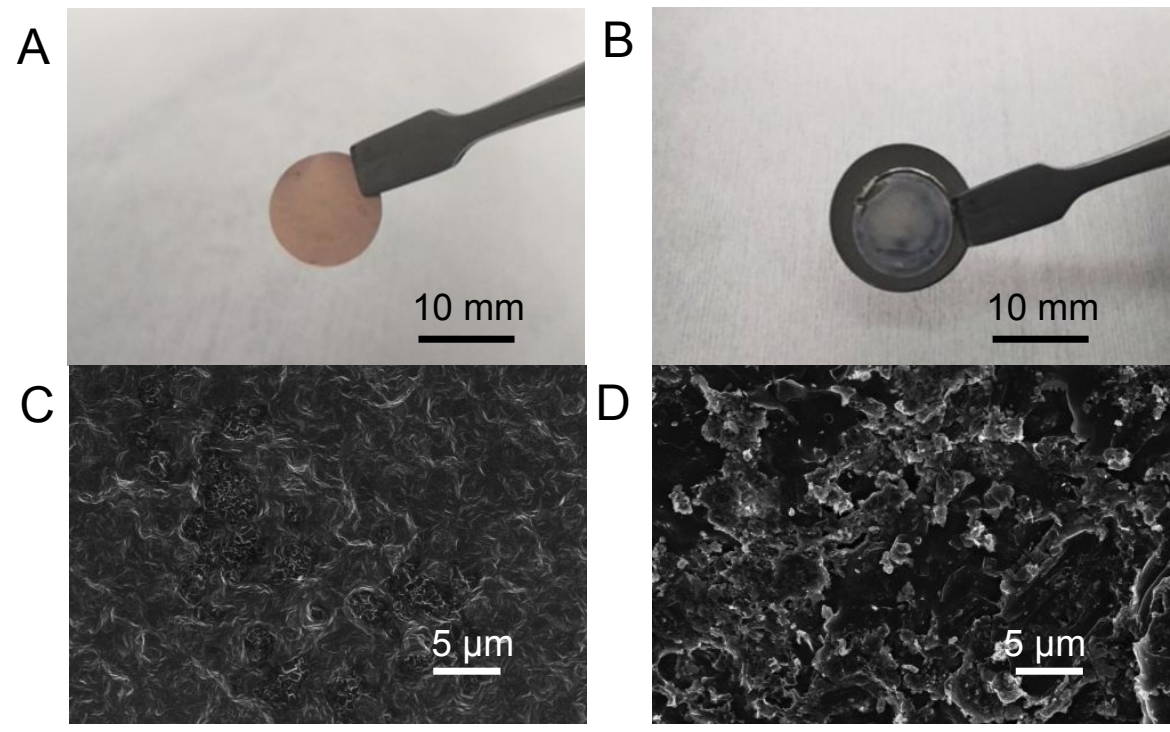

Figure S9. Optical images of the (A) $\mathrm{Cu}$ substrate (B) and LAGP pellet after depositing $1 \mathrm{mAh} \quad \mathrm{cm}^{-2} \quad \mathrm{Li}$ in $\mathrm{Li}|\mathrm{LAGP}| \mathrm{Cu}$ asymmetric cells an $\mathrm{d}$ the corresponding FE-SEM images of (C) $\mathrm{Cu}$ surface on (D) LAGP pellet after Li deposition. 

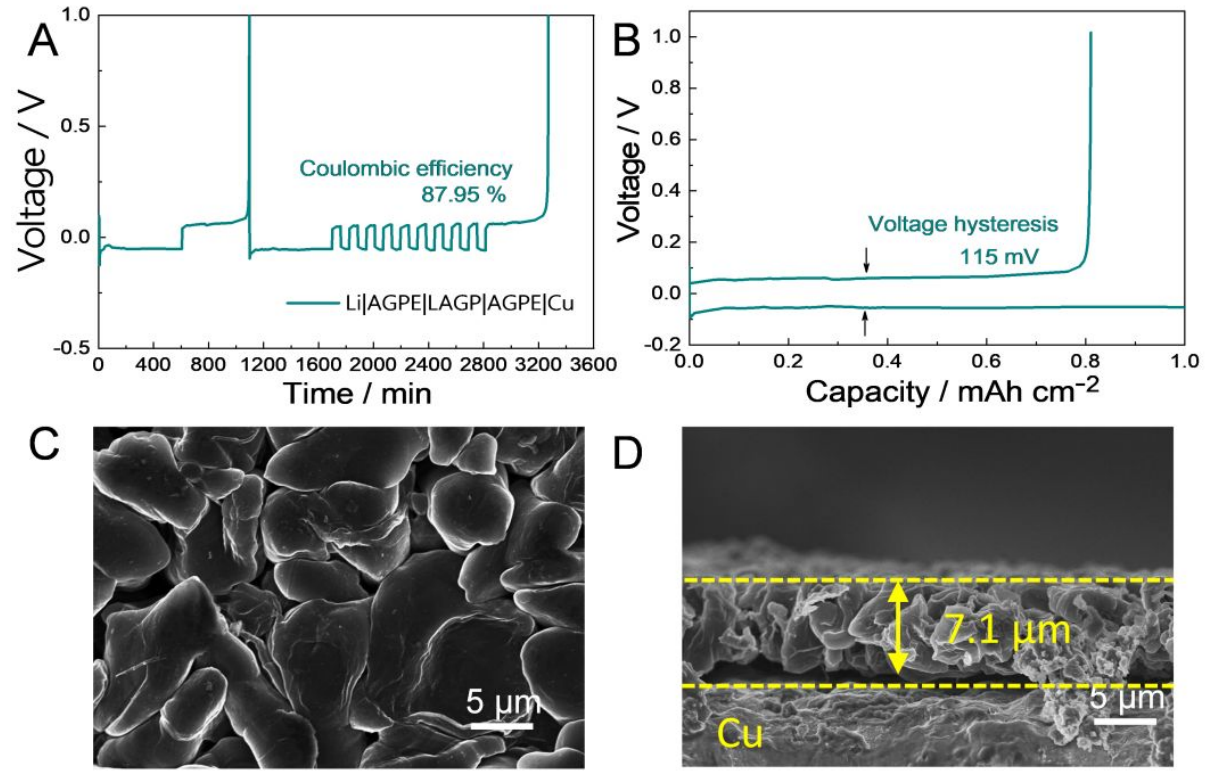

Figure S10. (A) Coulombic efficiency test of Li|AGPE|LAGP $\mid$ AGPE $\mid \mathrm{Cu}$ cell at 0.1 $\mathrm{mA} \mathrm{cm} \mathrm{cm}^{-2}$ and (B) the corresponding voltage profile during the Li plating-stripping process. (C) Top and (D) cross-sectional FE-SEM images of Li deposition obtained by plating $1 \mathrm{mAh} \mathrm{cm}^{-2} \mathrm{Li}$ on $\mathrm{Cu}$ substrate at $0.1 \mathrm{~mA} \mathrm{~cm}^{-2}$ in Li|AGPE|LAGP|AGPE $\mid \mathrm{Cu}$ cells. 

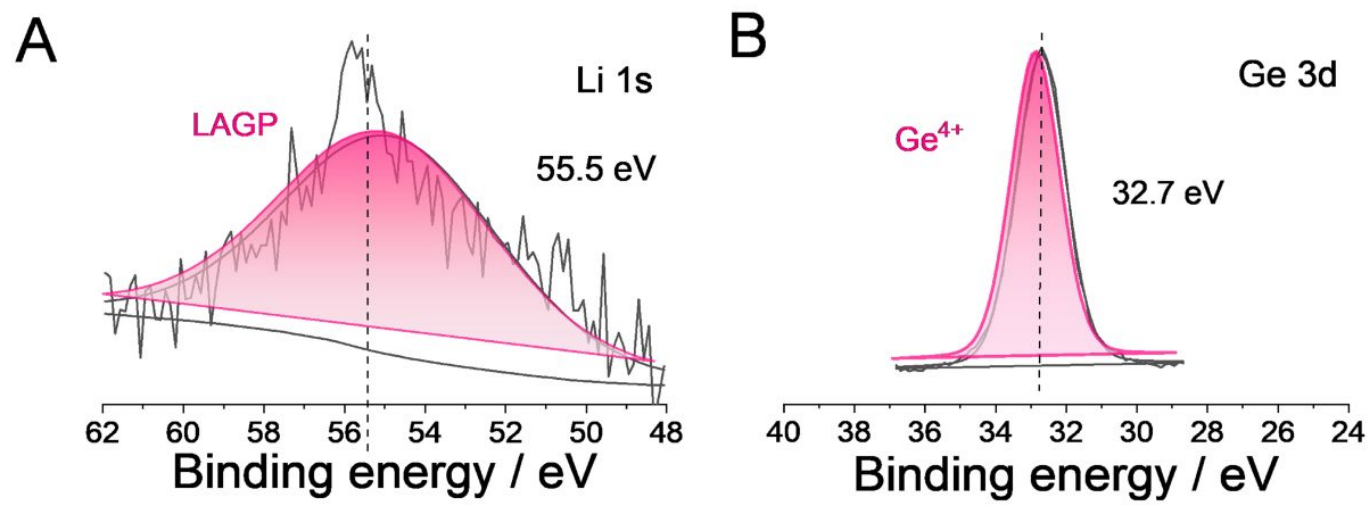

C
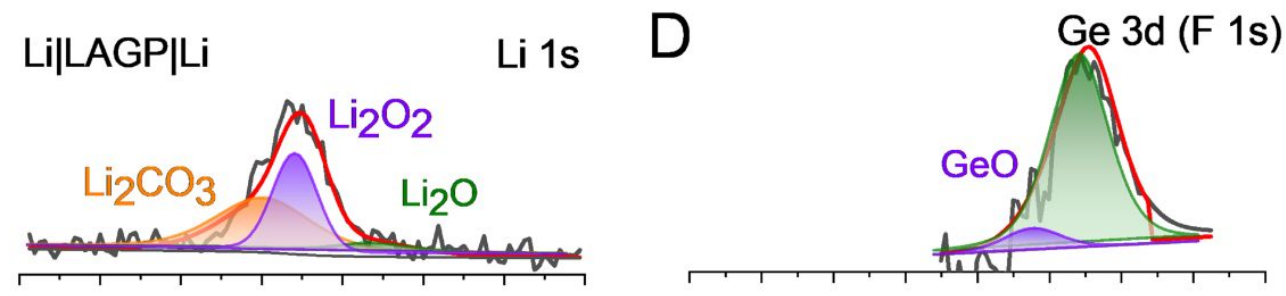

Li|AGPE|LAGP|AGPE|Li
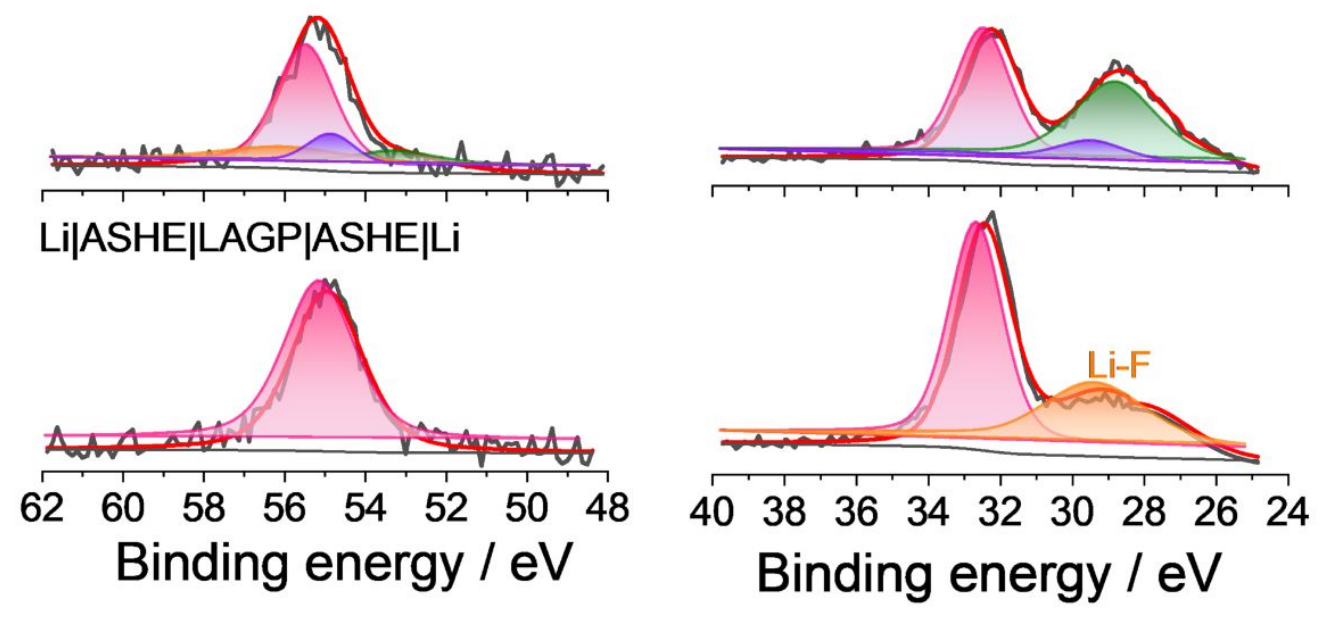

Figure S11. (A) Li 1s and (B) Ge 3d XPS spectra of the pristine LAGP pellet; (C) Li 1s and (D) Ge 3d XPS spectra of the LAGP pellet obtained from different Li|LAGP|Li symmetric cells after 10 cycles. 

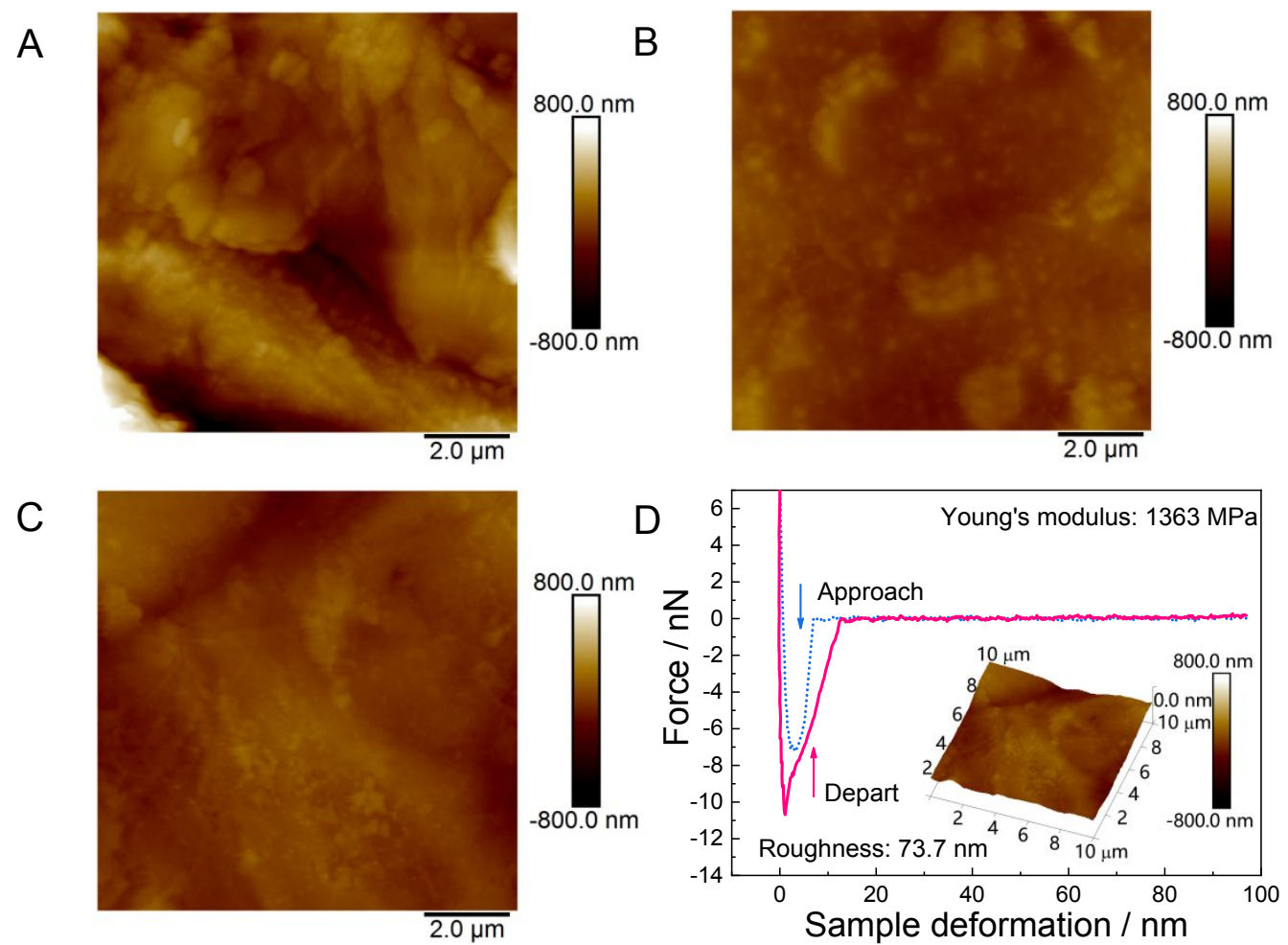

Figure S12. Two-dimensional AFM images of (A) LAGP-derived SEI (B) AGPE-derived SEI and (C) ASHE-derived SEI. (D) Force-displacement plots of AGPE-derived SEI. The corresponding 3D AFM scanning image of SEI layer is shown in inset. 


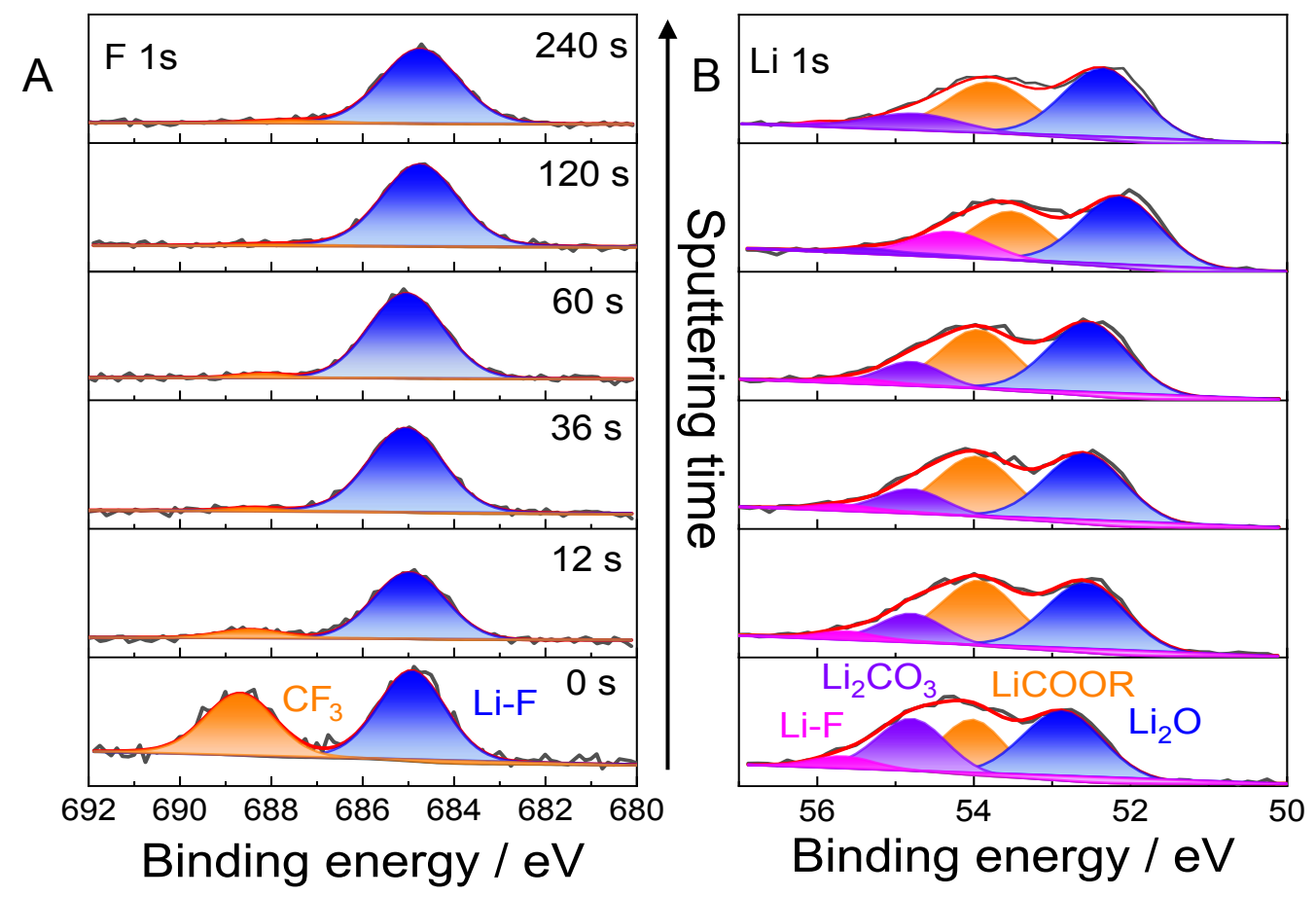

Figure S13. (A) F 1s and (B) Li 1s in-depth XPS spectra of the Li metal anode obtained from Li|AGPE|LAGP|AGPE|Li symmetric cell after 10 cycles. 


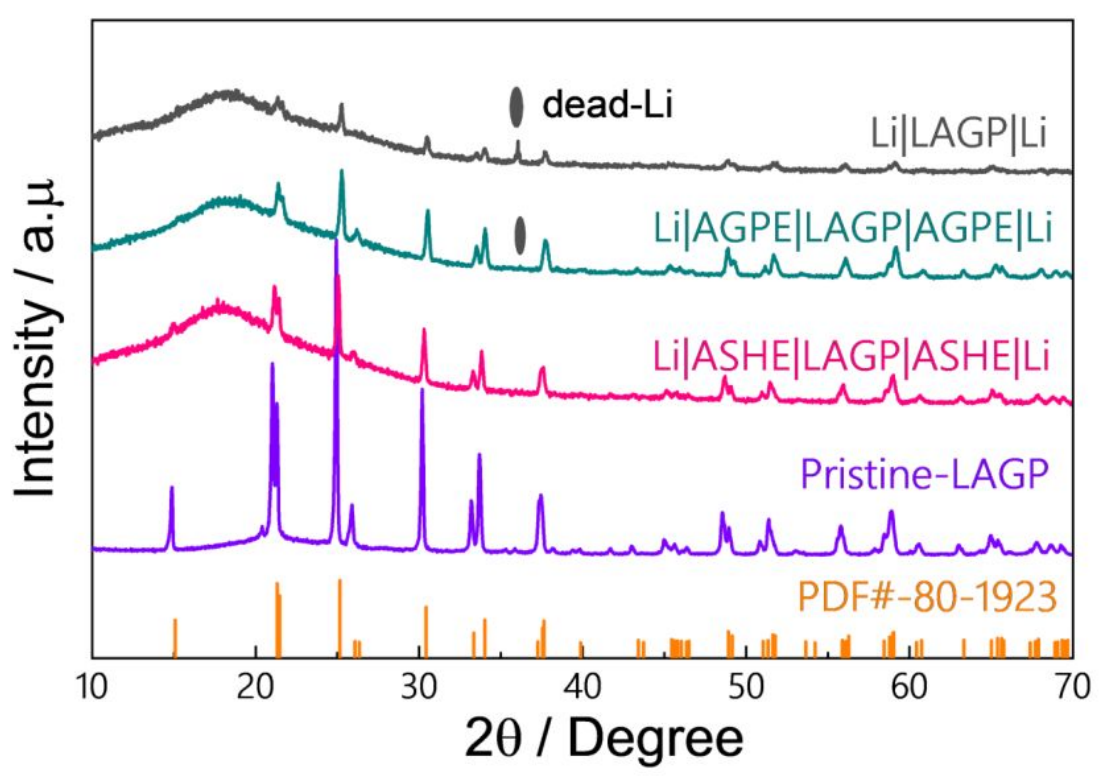

Figure S14. Ex situ XRD spectra of the pristine LAGP pellet and the LAGP pellet obtained from different Li||Li symmetric cells after 10 cycles. 


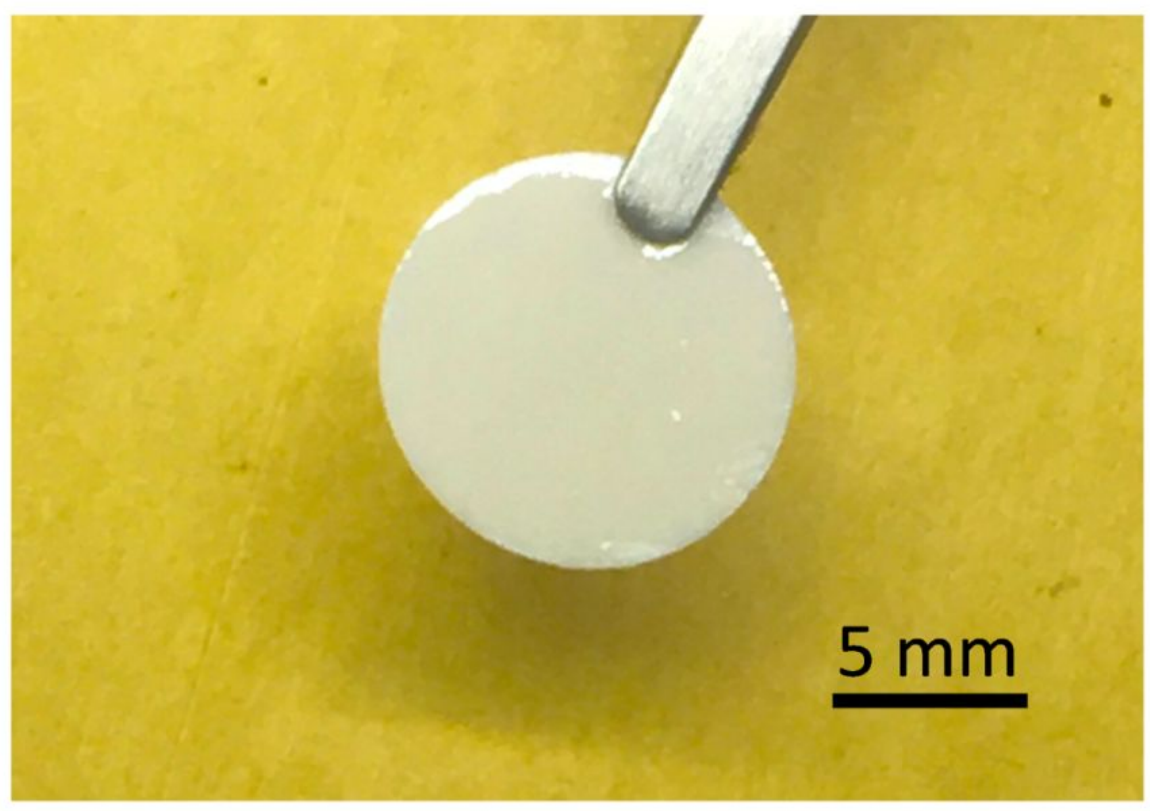

Figure S15. Optical image of the SHE-modified LAGP pellet. 

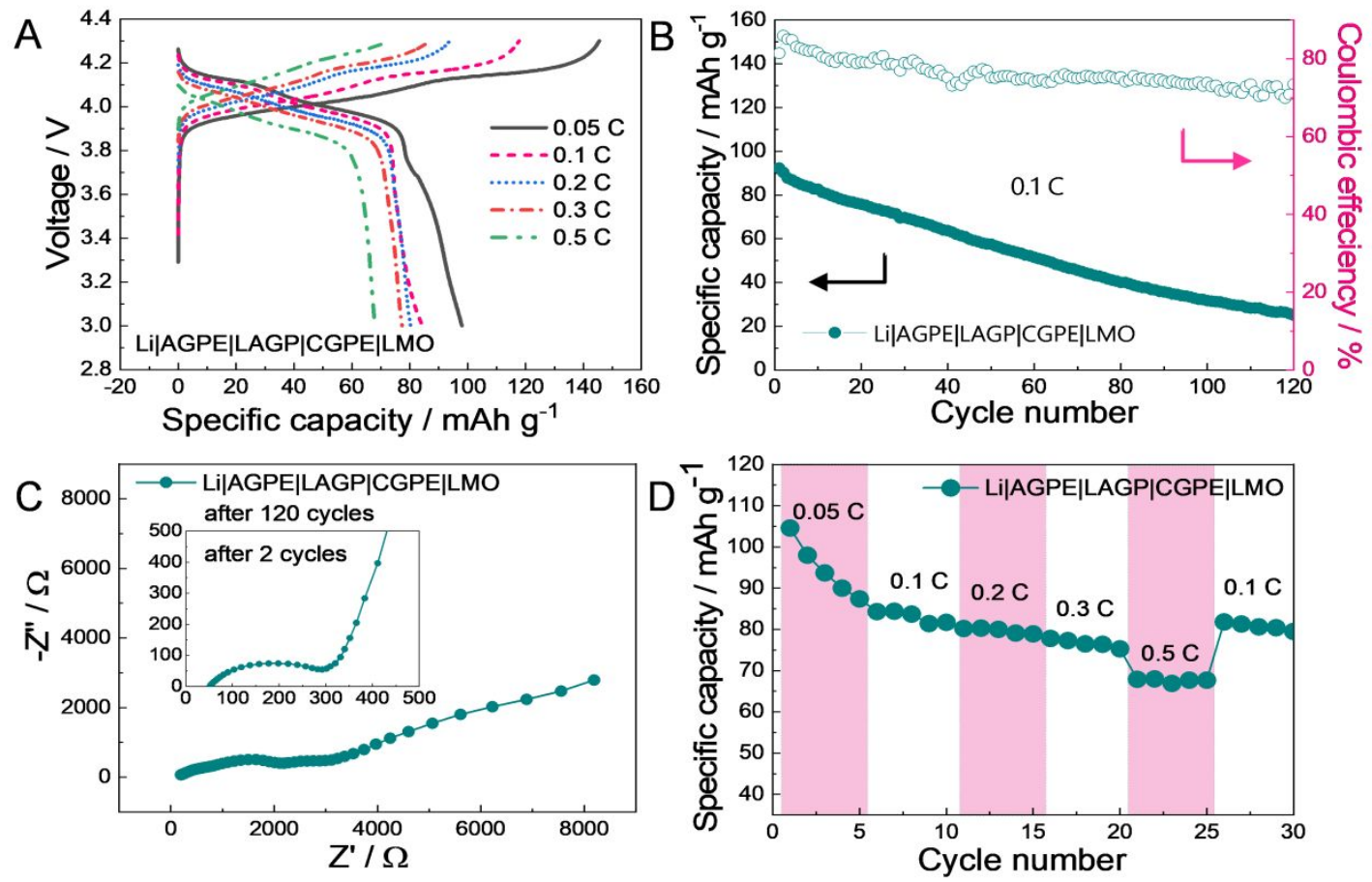

Figure S16. (A) Typical charge-discharge profiles at various rates and (B) cycling performance of $\mathrm{Li}|\mathrm{AGPE}| \mathrm{LAGP}|\mathrm{CGPE}| \mathrm{LMO}$ cells at 0.1 C. (C) EISs of Li|AGPE|LAGP|CGPE|LMO cells after 2 cycles (shown in inset) and 120 cycles at 0.1 C. (D) Rate performances of the Li|AGPE|LAGP|CGPE|LMO cell. 


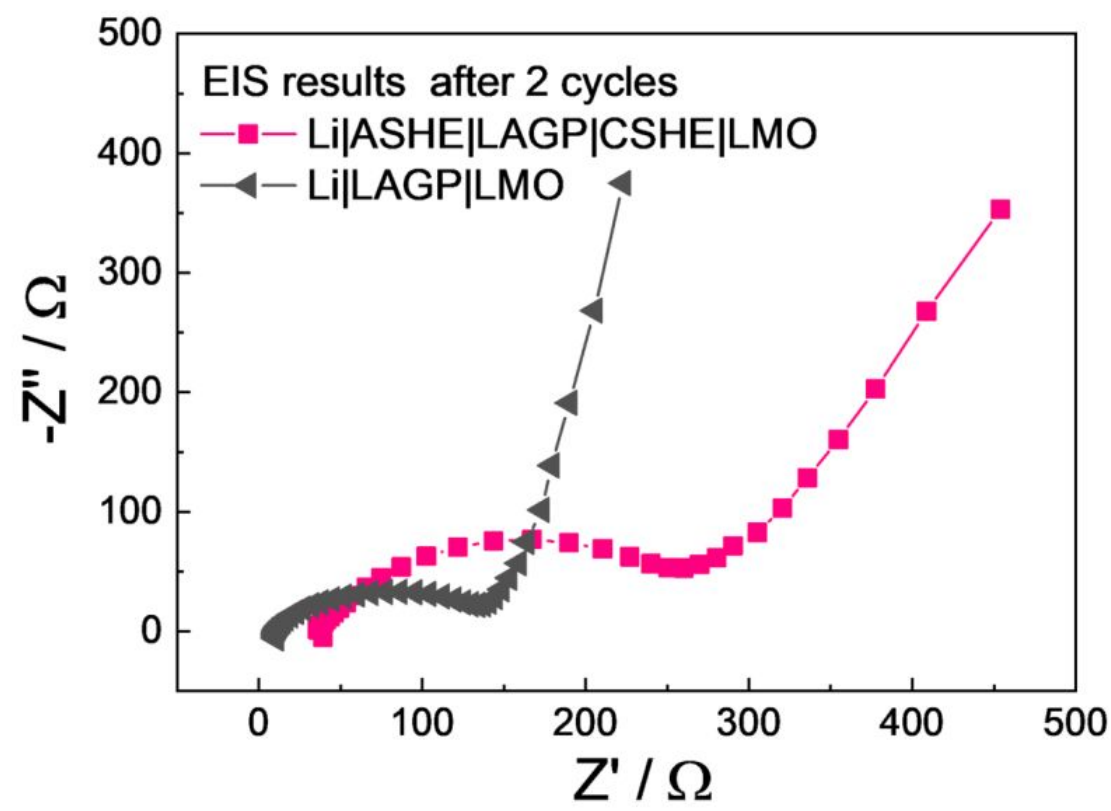

Figure S17. EISs of Li|LAGP|LMO and Li|ASHE|LAGP|CSHE|LMO cells after 2 cycles at $0.1 \mathrm{C}$. 


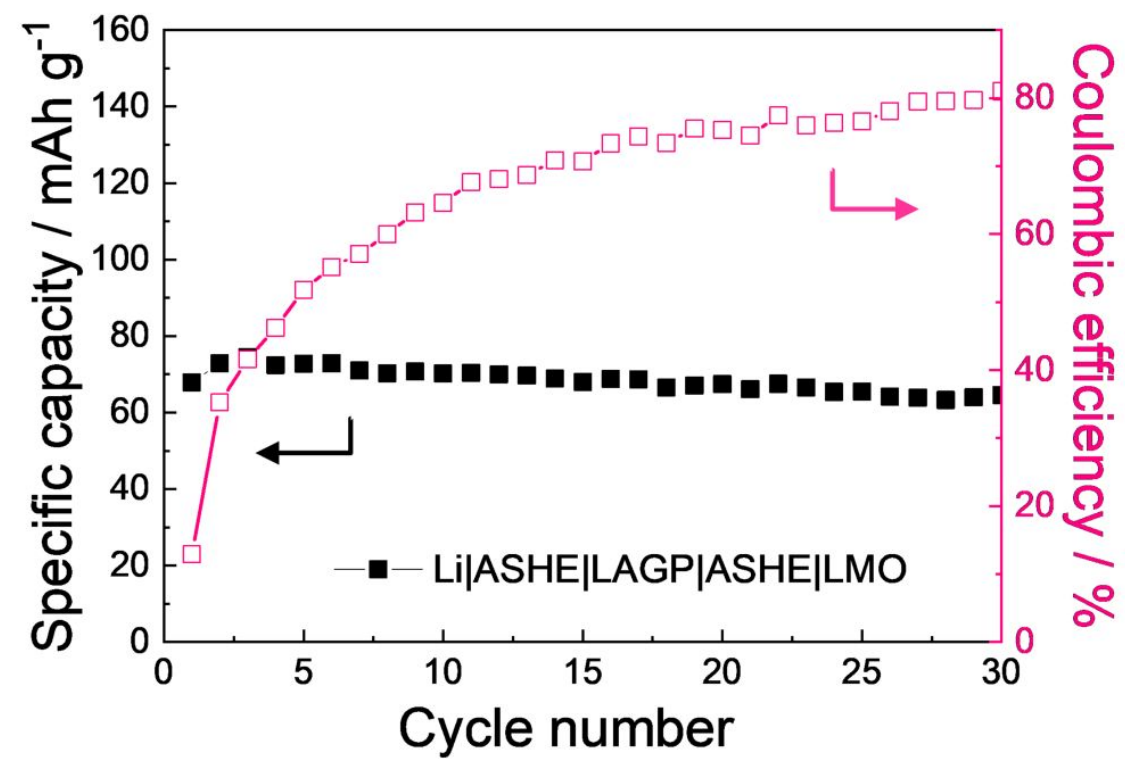

Figure S18. Cycling performance of Li|ASHE|LAGP|ASHE|LMO cell at $0.1 \mathrm{C}$. 

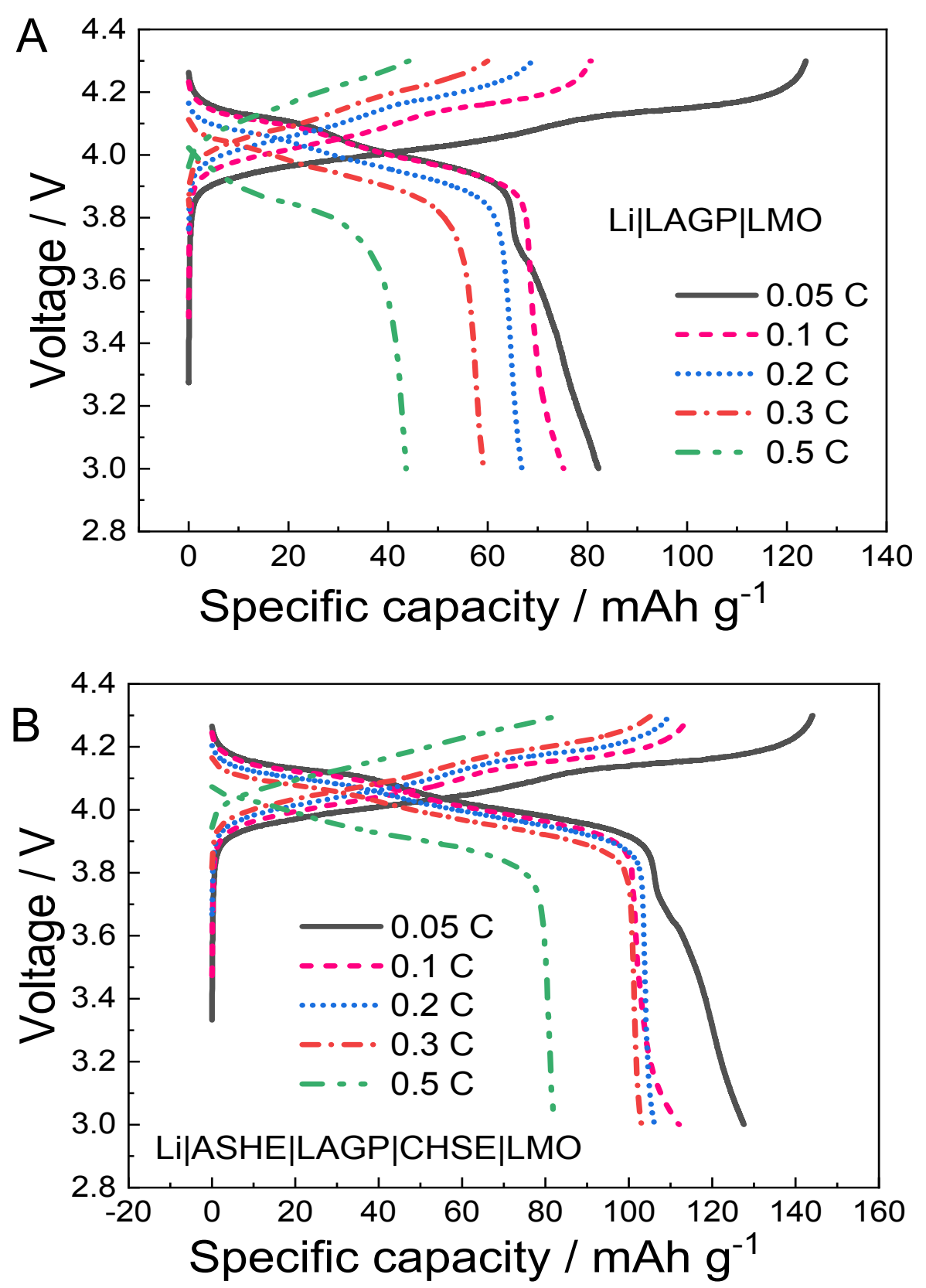

Figure S19. The charge-discharge curves of (A) Li|LAGP|LMO and (B) Li|ASHE|LAGP|CSHE|LMO cells at various rates. 


\section{REFERENCES}

(S1) Zhou, B.; He, D.; Hu, J.; Ye, Y.; Peng, H.; Zhou, X.; Xie, X.; Xue, Z., A flexible, self-healing and highly stretchable polymer electrolyte via quadruple hydrogen bonding for lithium-ion batteries. J. Mater. Chem. A 2018, 6 (25), $11725-11733$.

(S2) Adams, B. D.; Zheng, J.; Ren, X.; Xu, W.; Zhang, J.-G., Accurate Determination of Coulombic Efficiency for Lithium Metal Anodes and Lithium Metal Batteries. Adv. Energy Mater. 2018, 8 (7), 1702097.

(S3) Neese, F., Software update: the ORCA program system, version 4.0. WIREs Comput. Mol. Sci. 2018, 2017, e1327.

(S4) Grimme, S.; Antony, J.; Ehrlich, S.; Krieg, H., A consistent and accurate ab initio parametrization of density functional dispersion correction (DFT-D) for the 94 elements H-Pu. J. Chem. Phys. 2010, 132 (S5), 154104.

(S5) Grimme, S.; Ehrlich, S.; Goerigk, L., Effect of the damping function in dispersion corrected density functional theory. J. Comput. Chem. 2011, 32 (7), $1456-1465$.

(S6) Weigend, F.; Ahlrichs, R., Balanced basis sets of split valence, triple zeta valence and quadruple zeta valence quality for $\mathrm{H}$ to $\mathrm{Rn}$ : Design and assessment of accuracy. Phys. Chem. Chem. Phys. 2005, 7 (18), 3297-3305. 